Discrete Comput Geom 29:409-417 (2003)

DOI: $10.1007 /$ s00454-002-0774-3

\title{
An Improved Upper Bound for Leo Moser's Worm Problem
}

\author{
Rick Norwood and George Poole \\ Department of Mathematics, East Tennessee State University, \\ Johnson City, TN 37614, USA \\ \{norwoodr,pooleg\}@etsu.edu
}

\begin{abstract}
A worm $\omega$ is a continuous rectifiable arc of unit length in the Cartesian plane. Let $W$ denote the class of all worms. A planar region $C$ is called a cover for $W$ if it contains a copy of every worm in $W$. That is, $C$ will cover or contain any member $\omega$ of $W$ after an appropriate translation and/or rotation of $\omega$ is completed (no reflections). The open problem of determining a cover $C$ of smallest area is attributed to Leo Moser [7], [8]. This paper reduces the smallest known upper bound for this area from 0.275237 [10] to 0.260437 .
\end{abstract}

\section{Introduction, History, and Notation}

In 1966 Leo Moser published a set of open geometry questions, one of which is the following [7]-[9]: "What is the region of smallest area which will accommodate every planar arc of length one?" More explicitly, what is the size and shape of the planar region $C$ of minimum area so that any planar arc $\omega$ of fixed length 1 is a subset of that region, after suitable rotation and translation? The rotation and translation may be realized via a single isometry. The accommodation region is traditionally called a cover. A basic history of the worm problem may be found in [10]. Also see the web site below monitored by Steven Finch [2]: http://pauillac.inria.fr/algo/bsolve/constant/worm/worm.html. The basic facts are these: In the case $C$ is convex, the area of $C$ must lie between 0.21946 [14] and 0.27524 [10]. Moreover, a solution is known to exist, but it is not necessarily unique [5]. In the non-convex case, not much is known. According to Marstrand [6], the measure of a minimal cover must be positive. However, since a non-convex cover $C$ may contain large holes, it is not clear what a lower bound on the area (measure) might be in this case.

In this paper we construct a non-convex, simply connected region $C^{*}$ with area approximately equal to 0.260437 that will cover any planar arc $\omega$ of length 1 . In Section 2 we show that some current conjectures on convex covers are not true. In Section 3 we build two congruent "partial" covers $C$ and $C^{\prime}$ from which the desired non-convex, 
simply connected cover $C^{*}$ will be described. The argument that $C^{*}$ is a cover for $W$ is contained in Section 4. In Section 5 we present some remarks and insights regarding future solutions of the worm problem in the convex and non-convex case.

\section{Counterexamples and Insights}

In showing that some planar region $C$ (convex or not) is a candidate to be a cover for $W$, one should consider several very special arcs or "worms." If $C$ should fail to contain any one of these special arcs, $C$ cannot be a candidate. These special arcs are the straight arc of length 1, the $U$-shaped arc with three segments of length $\frac{1}{3}$ and convex hull a square (all sides of length $\frac{1}{3}$ ), the equilateral triangle arc with two segments of length $\frac{1}{2}$ and convex hull an equilateral triangle (all sides of length $\frac{1}{2}$ ), the Broad Worm of Schaer [13] whose diameter is maximum and equal in all directions, and the Z-shaped arc of Besicovitch [1].

In some early papers (e.g., [3] and [10]) it was thought that some special triangles might be convex covers. It was thought that the $30^{\circ}-60^{\circ}-90^{\circ}$ right triangle with hypotenuse $(6+2 \sqrt{3}) / 9$ and area less than 0.23942 might be a cover. However, Eric Ferguson [2] pointed out the rather easy observation that this triangle will not hold the U-shaped arc if it is slightly modified so that the outer two legs are kicked out to $91^{\circ}$ angles with the middle segment. It was also thought that the right triangle with sides of length 1 and $\frac{1}{2}$, and area equal to $\frac{1}{4}$ might be a cover. However, it can be easily shown that the one-angle arc whose convex hull is a triangle with sides of length 0.48 and 0.52 , and with included angle of measure $72^{\circ}$, cannot be covered by this special right triangle.

In discovering the one-angle arc that could not be covered by the $30^{\circ}-60^{\circ}-90^{\circ}$ triangle with area $\frac{1}{4}$, we became convinced that left-right symmetry will be required of either a convex or non-convex minimal planar cover for $W$. That is, a one-angle worm and its reflection (not permitted of its cover) must be covered. Consequently, we turned our attention to a left-right symmetric region and abandoned the convexity requirement. However, we insist that the region be simply connected. A planar region is simply connected if and only if its fundamental group contains only one element [12]. That is, the region is pathwise connected and contains no holes. Finally, there are two important observations worth noting. Both observations represent information for would-be problem solvers of the Worm Problem and the first is used in Section 4 below.

Observation 2.1. Every worm lies within $\varepsilon$ of a shorter worm that does not cross itself. That is, for all $\varepsilon$ greater than zero there is a shorter non-self-intersecting worm such that every point of the worm lies within $\varepsilon$ of a point on the non-self-intersecting worm.

Observation 2.2. Every worm lies within $\varepsilon$ of a polygonal worm.

The cover $C^{*}$ to be constructed in the next section is a simply connected set. Consequently, since both observations above are true for all positive $\varepsilon$, in order to show $C^{*}$ is a cover for $W$, it is sufficient to show that every polygonal, non-self-intersecting worm is covered by $C^{*}$. The proof is straightforward point-set topology. 


\section{Construction of Two Partial Covers $C$ and $C^{\prime}$}

The idea for this construction came from Meir's semicircle cover [15] and Poole and Gerriets' rhombus cover [3], [11]. In a standard Cartesian plane, place the straight arc along the $x$-axis so that its midpoint is at the origin, hence its endpoints are at $\left(-\frac{1}{2}, 0\right)$ and $\left(\frac{1}{2}, 0\right)$. Label these two endpoints $\mathrm{L}$ (left) and R (right), respectively. These two points remain fixed throughout the discussion. Given a number $0<t<\frac{1}{4}$, we wish to describe the boundary of the cover $C(t)$ with respect to two other points, $\mathrm{T}$ (top) on the $y$-axis with coordinates $(0, t)$ and $\mathrm{B}$ (bottom) on the $y$-axis below the origin with coordinates $(0, b)=\left(0, t-\frac{1}{2}\right)$. We wish to construct a region $C(t)$ as a function of $t$, with a boundary composed of two parts: The top boundary $\tau$ will be the arc of a circle passing through $\mathrm{L}, \mathrm{T}$, and $\mathrm{R}$, while the bottom boundary $\beta$ connecting $\mathrm{L}$ and $\mathrm{R}$ will be the union of two parabolic arcs that are mirror images of each other in the $y$-axis. First, we construct a circular arc $\tau$ passing through $\mathrm{L}, \mathrm{T}$, and $\mathrm{R}$ as follows: Its center is $(0, c)$ where $c=\left(t^{2}-\frac{1}{4}\right) / 2 t$, and its radius is $r=t-c$. Second, we construct the right half of the lower boundary $\beta$ of $C(t)$ connecting $\mathrm{B}=(0, b)$ to $\mathrm{R}=\left(\frac{1}{2}, 0\right)$ as follows: The right half of $\beta$ is the locus of points $(x, y)$ such that the sum of the distance $d_{1}$ from the $y$-axis to $(x, y)$ and the distance $d_{2}$ from $(x, y)$ to $\tau$ along the radius from point $(0, c)$ to $\tau$ is exactly $\frac{1}{2}$. Under these conditions the equation of the top boundary $\tau$ is $x^{2}+(y-c)^{2}=r^{2}\left(-\frac{1}{2} \leq x \leq \frac{1}{2}\right)$ and the equation of the right half of the bottom boundary $\beta$ is $y=\sqrt{2 w x+w^{2}}+c\left(0 \leq x \leq \frac{1}{2}\right)$ where $w=r-\frac{1}{2}$. The left half of $\beta$ is the mirror image of the right half of $\beta$ in the $y$-axis.

Properties of $C(t)$. Suppose $\omega$ is a member of $W$ with midpoint $m$ which divides $\omega$ into two half-arcs, $\alpha$ and $\gamma$. We position $\omega$ so that it lies entirely above the boundary $\beta$ of $C(t)$ with $m$ on the $y$-axis. We slide $\omega$ down, keeping $m$ on the $y$-axis, until both halves, $\alpha$ and $\gamma$, are contiguous with $\beta$, or, if in all possible orientations of $\omega$ only one half is able to touch, say $\gamma$, then until $\gamma$ touches. Here we are using "contiguous with" to mean "touches, but does not cross." Furthermore, in all possible orientations (either both $\alpha$ and $\gamma$ are contiguous with $\beta$, or only $\gamma$ is contiguous with $\beta$ ), select one for which $m$ is as low as possible, or in other words, so the distance from B to $m$ is smallest. We refer to this orientation as the Minimal Positioning Scheme (MPS). By the way $C(t)$ was constructed, for every $0<t<\frac{1}{4}$ neither $\alpha$ nor $\gamma$ can first touch $\beta$ and then "escape" through the top boundary $\tau$. In order to "get out" (not be covered), $\alpha$ or $\gamma$ must travel first to the top boundary $\tau$ and then return to the bottom boundary $\beta$ using distance less than $\frac{1}{2}$. We call this condition Property $A$, to which we shall refer later.

Now, for infinitely many values of $t, 0<t<\frac{1}{4}$, it is the case that either of the $\operatorname{arcs} \alpha$ or $\gamma$ could "travel" from $m$ to the top boundary $\tau$ and back to the bottom boundary $\beta$ in a distance less than $\frac{1}{2}$ (and, hence, could escape). In this case, $C(t)$ would not cover $\omega$. So we drew the boundary of $C(t)$ for values of $0<t<\frac{1}{4}$. There exists a value of $t$ such that when $m$ is on or below the origin, the distance from $m$ to the boundary $\tau$ and back to $\beta$ always equals or exceeds $\frac{1}{2}$. Therefore, such worms, if they satisfy MPS, cannot escape. A straightforward continuity argument shows that a minimum value of $t$ exists. The approximate minimum value of $t$, with the property described above, is 0.1527985 . Hence, $b=-0.3472015$. 


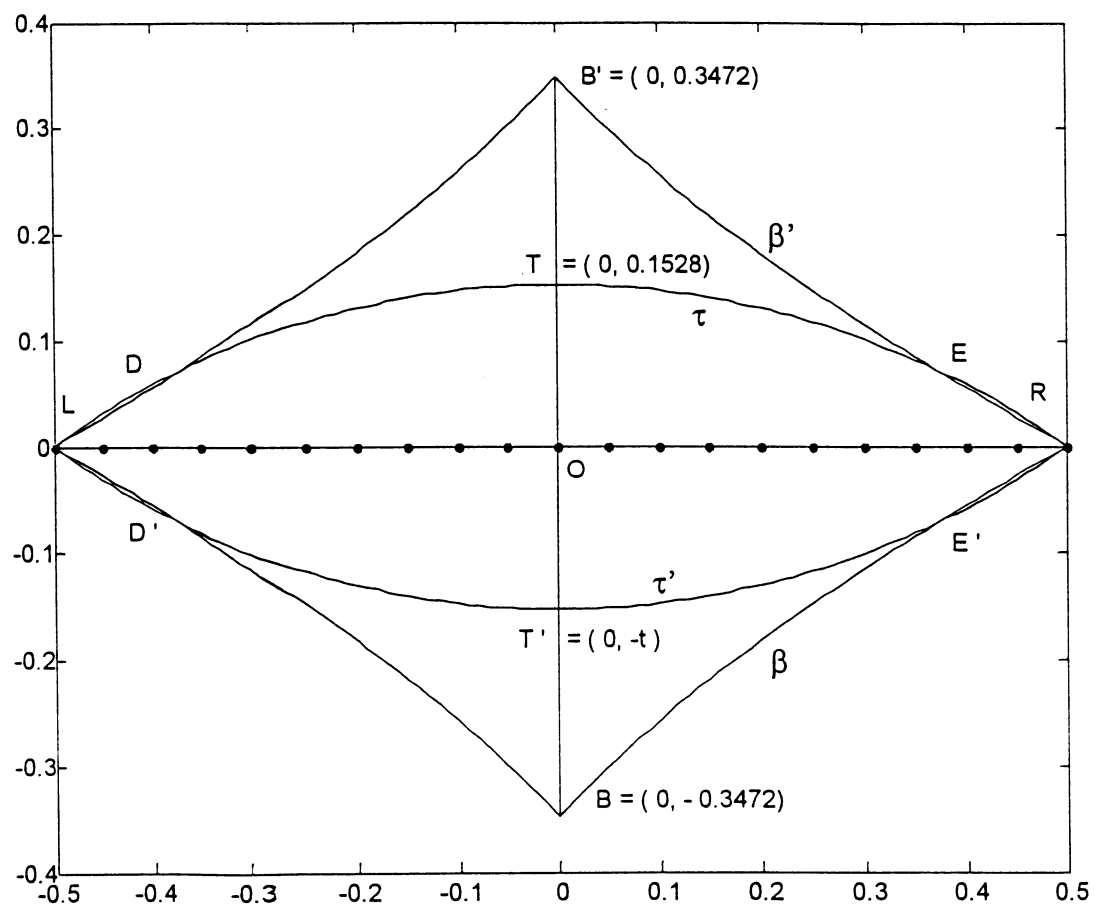

Fig. 3.1

Now we consider the cover candidate $C(t=0.1527985)$, simply called $C$. Suppose $\omega$ is in an MPS orientation. It is now the case that if $m$ lies at or below the origin, $\omega$ is covered by $C$. That is, neither $\alpha$ nor $\gamma$ can touch $\beta$ and escape beyond the boundary of $C$, either by traveling first to $\beta$ and then to $\tau$, or by traveling first to $\tau$ and then to $\beta$. We call this condition Property $B$, to which we shall refer later. The area of $C$ is 0.2596605 , correct to seven places. We believe $C$ covers all elements of $W$. However, to facilitate the proof below, we modify $C$ slightly in order to obtain the cover $C^{*}$. Hereafter, $C$ is the cover $C(0.1527985)$ with key boundary points $\mathrm{L}, \mathrm{T}, \mathrm{R}$, and $\mathrm{B}$.

Let $C^{\prime}$ be the reflection of $C$ about the $x$-axis with key boundary points $\mathrm{L}^{\prime}=\mathrm{L}, \mathrm{T}^{\prime}=$ $(0,-t), \mathrm{R}^{\prime}=\mathrm{R}$, and $\mathrm{B}^{\prime}=(0,0.3472015)$ as shown in Fig. 3.1 (coordinates are printed to four places). Let $\tau^{\prime}$ and $\beta^{\prime}$ denote the boundary portions of $C^{\prime}$ that represent the reflections of $\tau$ and $\beta$ about the $x$-axis. Let $\tau(r)$ and $\beta^{\prime}(r)$ represent the right halves of the arcs $\tau$ and $\beta^{\prime}$, respectively. Note that there are two points of intersection for these two arcs, one at $\mathrm{R}=\left(\frac{1}{2}, 0\right)$ and the other at $\mathrm{E}=(0.370455,0.072453)$. This second point is correct to the indicated digits. Furthermore, between $\mathrm{E}$ and $\mathrm{R}, \tau$ lies above $\beta^{\prime}$. $\mathrm{E}^{\prime}$ is the reflection of $\mathrm{E}$ about the $x$-axis onto $\beta$, that is, $\mathrm{E}^{\prime}=(0.370455,-0.072453)$. Let $\mathrm{D}^{\prime}$ be the reflection of $\mathrm{E}^{\prime}$ about the $y$-axis onto $\beta$. Finally, let $\mathrm{D}$ be the reflection of $\mathrm{D}^{\prime}$ about the $x$-axis onto $\tau$. $\mathrm{D}$ is also the reflection of $\mathrm{E}$ about the $y$-axis onto $\tau$.

Using Fig. 3.1 as a reference, let $C^{*}$ denote the region whose top boundary is $\tau$ as described above, passing through the points L, D, T, E, and R. Hereafter, the boundary $\tau$ is referred to as the top of $C^{*}$. The bottom boundary of $C^{*}$ is $\beta$ between the points $\mathrm{D}^{\prime}$ 
and $\mathrm{E}^{\prime}$. The bottom boundary of $C^{*}$ is $\tau^{\prime}$ between the pairs of points $\mathrm{L}$ and $\mathrm{D}^{\prime}$, and $\mathrm{E}^{\prime}$ and R (referred to later as "bubbles"). Hereafter, this lower boundary of $C^{*}$ is referred to as the bottom of $C^{*}$. This determines the region $C^{*}$ uniquely, and $C^{*}$ is the desired cover. The area of the region $C^{*}$ is less than 0.2604370 , a little more than the area of $C$, which is 0.2596605 .

\section{The Main Theorem}

The cover $C^{*}$ described in Section 3 is a non-convex, simply connected cover for all worms of length 1 .

Theorem. The cover $C^{*}$ with boundary points $\mathrm{L}, \mathrm{D}, \mathrm{T}, \mathrm{E}, \mathrm{R}, \mathrm{E}^{\prime}, \mathrm{B}, \mathrm{D}^{\prime}$, and $\mathrm{L}$ has area equal to 0.2604370 (correct to seven places) and is a cover for $W$.

Proof. In this proof we show that every non-self-intersecting worm is covered. By Observation 2.1 above, this shows that every worm is covered. Let $C^{* *}$ be the figure obtained by rotating $C^{*} 180^{\circ}$ about the origin. Call $C^{*}$ the original cover and call $C^{* *}$ the inverted cover. We refer to the top and bottom of $C^{*}$, as described above, and to the "top" and "bottom" of $C^{* *}$, noting that because of the rotation, the "bottom" is above the "top." In fact, throughout this proof we strongly depend on the observation that the bottom of $C^{*}$ is on or below the "top" of $C^{* *}$ which is on or below the top of $C^{*}$ which is on or below the "bottom" of $C^{* *}$ (see Fig. 3.1).

Let $\omega$ denote a non-self-intersecting worm of length 1 with midpoint $m$ that divides the worm into two equal subarcs, $\alpha$ and $\gamma$, each of length $\frac{1}{2}$.

Recall: If $m$ lies on the origin, or between the origin and the vertex of a cover (either $C^{*}$ or $\left.C^{* *}\right)$, then $\omega$ cannot first touch the bottom and then cross the top of that cover (Property A). So if one side of the worm touches the bottom of that cover, then that side of the worm is covered by that cover (Property B). If $\omega$ is positioned in MPS, then $\omega$ cannot first touch the bottom and then cross the top of either cover. We now consider two main cases and within each case, several subcases.

Case 1. The first case we consider is where only one-half of the worm ever touches the bottom of a cover. In other words, in this case, when we lower the worm toward the bottom of the cover, there is one fixed half that always touches first, no matter how the worm is oriented. Call this half $\gamma$ and the other half $\alpha$. If $\gamma$ touches the bottom of a cover without crossing, $\alpha$ does not touch the bottom of that cover. Call this Property $C$.

We position the worm so that of all orientations with $m$ on the $y$-axis, $m$ is as close to the vertex of the cover as possible (MPS). We prove by contradiction that a worm that satisfies MPS is covered. If the worm is not covered, then either $\alpha$ or $\gamma$ must escape. In Case $1 \mathrm{~A}$ we consider whether $\gamma$ can escape, and show that it is covered.

Case 1A. Suppose, by way of contradiction, that $\gamma$ gets out through the top. $\gamma$ must touch the bottom, or the worm could be lowered (contradicting MPS). By Property A, $\gamma$ must first cross the top of the original cover $C^{*}$, then touch the bottom of the original cover $C^{*}$. By Property B, $m$ must be above the origin. If $\gamma$ did not cross the "bottom" 
of $C^{* *}$, we could move $\gamma$ up until it just touched the "bottom" of $C^{* *}$ (or, if it already touches "bottom," leave it alone). In moving $\gamma$ up, $\alpha$ does not touch the "bottom" of $C^{* *}$, by Property C. Now $m$ lies strictly above the origin and below the vertex of the inverted cover $C^{* *}$, contradicting MPS.

Therefore $\gamma$ does cross both the top of the original cover and the "bottom" of $C^{* *}$, then returns to touch the bottom of $C^{*}$. However, then the worm can be moved down so that $\gamma$ just touches the "bottom" of $C^{* *}$, and then crosses the bottom of $C^{*}$. On its way from the "bottom" of $C^{* *}$ to the bottom of $C^{*}, \gamma$ must cross the "top" of $C^{* *}$. This contradicts Property A. Therefore $\gamma$ is covered.

In Case 1B we will show that $\alpha$ is also covered.

Case 1B. Suppose, by way of contradiction, that when the worm satisfies MPS in the original cover $C^{*}, \alpha$ gets out through the top at point $a$ on $\alpha$. We have already shown that $\gamma$ does not get out, and so we can move the worm up (or leave it fixed) so that $\gamma$ touches the "bottom" of $C^{* *}$ (after which, point $a$ on $\alpha$ is either on or above the top of $C^{*}$ ).

After this move, $\gamma$ is either inside or outside the inverted cover. We call these Subcases $1 \mathrm{~B}$ (i) and $1 \mathrm{~B}$ (ii) and consider each in turn, showing that each of these possibilities will lead either to the worm being covered or to a contradiction of Property C.

Subcase 1B(i). First we consider the possibility that $\gamma$ is inside the inverted cover. If $\alpha$ is also inside the inverted cover, then the worm is covered. If, on the other hand, $\alpha$ gets out of the inverted cover $C^{* *}$, it must cross the "top" of $C^{* *}$ at some point $b$. We know $\alpha$ cannot cross the "bottom" of $C^{* *}$ because in this case, when we lower the worm into any cover, $\gamma$ always touches first. Since $\gamma$ does not cross the "bottom" of $C^{* *}$, neither does $\alpha$. Now let $\mathrm{L}_{1}$ be the line tangent to the top of the original cover $C^{*}$ at the original position of $a$. When we raise the worm, also raise $\mathrm{L}_{1}$. Since $\gamma$ was covered by the original cover, it does not cross $\mathrm{L}_{1}$, which lies outside the original cover. $\gamma$ does not cross the part of $\alpha$ between $a$ and $b$ because the worm is non-self-intersecting. Let $\mathrm{L}_{2}$ be the line tangent to the "top" of the $C^{* *}$ at $b . \gamma$ does not cross $\mathrm{L}_{2}$, because in this case $\gamma$ is inside the inverted cover $C^{* *}$. The part of $\alpha$ between $a$ and $b$ separates the region between $\mathrm{L}_{1}$ and $\mathrm{L}_{2}$ into two parts, and since the worm $\omega$ is non-self-intersecting, $\gamma$ lies entirely in one of those parts. We now rotate the worm, keeping $m$ on the $y$-axis, so that a line through $a$ and $b$ would be horizontal, and so that every point of $\gamma$ lies on or above some point of $\alpha$ between $\mathrm{L}_{1}$ and $\mathrm{L}_{2}$. Then we adjust the worm until it just touches the bottom of the original cover. It is clear that in this position, $\alpha$ will touch first, contradicting the assumption of Case 1.

Subcase 1B(ii). Now we consider the possibility that $\gamma$ is not inside the inverted cover. In other words, after we have raised the worm so that $\gamma$ just touches the "bottom" of $C^{* *}$ at $c$, we consider whether or not it is possible for $\gamma$ to escape from the "top" of $C^{* *}$ at some point $d$. If this happens, then by Property A, $\gamma$ goes first from $m$ to $d$ and then goes from $d$ to $c$. Also, by Property $\mathrm{B}, m$ lies below the origin (because the origin lies between $m$ and the vertex of the inverted cover $C^{* *}$ ).

Assume, without loss of generality, that $\alpha$ lies to the left of the part of $\gamma$ between $c$ and $d$. To see that this assumption is reasonable, note that $\alpha$ cannot pass directly above $c$ nor directly below $d$, because if it did, then we could raise or lower the worm so that $\alpha$ 
touched the bottom of a cover before $\gamma$ (contrary to assumption). Let $x$ denote the length of the part of $\gamma$ between $m$ and $d$. Let $y$ denote the length of the part of $\gamma$ between $d$ and $c$. Let $z$ denote the length of the part of $\gamma$ beyond $c$. Then $x+y+z=\frac{1}{2}$ and $\gamma$ lies inside the union of the three regions whose boundaries are two ellipses and a circle. We call these three regions the upper arm, forearm, and hand, respectively. The first ellipse has foci $m$ and $d$ and major axis of length $x$; the second ellipse has foci $d$ and $c$ and major axis of length $y$; and the circle has center $c$ and radius $z$. We denote the union of the three regions, upper arm, forearm, and hand, by $\Gamma$.

By Property A, no worm with center $m$ can touch the bottom of the original cover and then reach $c$. Therefore $\Gamma$, every point of which is a possible point of some worm with $m, d, c$ and $x, y, z$ as defined above, is above the bottom of the original cover $C^{*}$. Now we lower the worm, and $\Gamma$ with it, until $\Gamma$ just touches the bottom of the original cover. Since every point in $\Gamma$ is a possible location of a worm with center $m$, and since $m$ is below the origin, $\Gamma$ is now covered by the original cover.

In this position, suppose $\alpha$ can escape through the top of the original cover at some point $p$. If so, then $p$ lies on the top of the original cover $C^{*}$ to the left of the forearm of $\Gamma$ and outside the convex hull of $\Gamma$ (since $\Gamma$ is under the convex top of the original cover). Now we rotate the worm about $m$ until $p$ lies directly above $c$. Then we raise the worm. The point $p$ touches the "bottom" of $C^{* *}$ first, a contradiction.

This completes the proof that a worm, if it has the property that only one half ever touches the bottom of a cover when the center $m$ is on the $y$-axis, is covered when it satisfies MPS.

Case 2. Case 2 is the case where for some orientation $\alpha$ touches the bottom of the cover first and for another orientation $\gamma$ touches the bottom of the cover first. By continuity there is an orientation where both $\alpha$ and $\gamma$ touch, and furthermore there is at least one orientation in which both $\alpha$ and $\gamma$ touch the bottom of the original cover so that $m$ is as low as possible among all orientations where both $\alpha$ and $\gamma$ touch (MPS). We prove by contradiction that the worm that satisfies MPS is covered.

We call a point where $\alpha$ touches the bottom of the original cover $e$; and we call a point where $\gamma$ touches the bottom of the original cover $c$.

Suppose, by way of contradiction, that the worm in this position is not covered. Then by Property B, $m$ lies above the origin, and by Property A at least one side of the worm, we may assume it is $\gamma$, gets out by first crossing the top of the original cover at $d$ and then touching the bottom of the original cover at $c$, while $\alpha$ touches the bottom of the original cover at $e$. Assume, without loss of generality, that $e$ lies to the left of $c$.

In this position the worm $\omega$ must lie entirely on or below the "bottom" of $C^{* *}$. For if the worm $\omega$ crossed the "bottom" of the inverted cover $C^{* *}$, it could not also touch the bottom of the original cover $C^{*}$, because in doing so it would have to touch or cross the "top" of $C^{* *}$ with the center $m$ between the origin and the vertex of the inverted cover $C^{* *}$, contradicting Property B. In fact, as an additional note, $\omega$ cannot even touch the "bottom" of $C^{* *}$.

Now we raise the worm until it just touches the "bottom" of the $C^{* *}$.

Both sides of the worm cannot touch, because we are considering the case where, when the worm is as low as possible in a cover with both sides touching, the center is on the other side of the origin from the vertex of that cover. 
We will arrive at a contradiction by rotating and sliding the worm until both sides of the worm do touch the "bottom" of $C^{* *}$ while keeping $m$ between the origin and the vertex $\mathrm{B}^{\prime}$ of $C^{* *}$.

Case 2A. First, we consider what happens if $\gamma$ just touches the "bottom" of $C^{* *}$. Keeping $m$ on the $y$-axis and keeping the worm just touching the "bottom" of $C^{* *}$, rotate the worm clockwise. $\gamma$ will stay in contact with the "bottom" of $C^{* *}$ until eventually $\alpha$ swings around and touches the "bottom" of $C^{* *}$. To arrive at a contradiction, we show that when this happens $m$ is still between the origin and $\mathrm{B}^{\prime}$.

We now return the worm to its original position, and construct $\Gamma$ just as in Case 1B(ii) above. Now raise the worm until $\Gamma$ just touches the "bottom" of $C^{* *}$. We consider what happens as we rotate both the worm and $\Gamma$ clockwise about $m$, keeping $m$ on the $y$-axis and moving $m$ up or down as necessary to keep $\Gamma$ contiguous with the "bottom" of $C^{* *}$. The elliptical regions upper arm and forearm slide along the "bottom" of $C^{* *}$ until some point of $\alpha$ touches the "bottom" of $C^{* *}$. If no other point of $\alpha$ touches, certainly $e$ touches, since by the same argument as in Case $1 \mathrm{~B}(\mathrm{ii}), e$ lies on or outside the convex hull of $\Gamma$. There is then, by continuity, some rotation no greater than that, where both $\alpha$ and $\gamma$ touch. If $m$ still lies above the origin, we have our contradiction of MPS.

To see that $m$ still lies above the origin, we consider some distances. The shortest distance from the origin to the "bottom" of $C^{* *}$ is more than 0.2645 , a numerical computation. For $m$ to be below the origin while $\Gamma$ is touching the "bottom" of $C^{* *}$, the major axis of the upper arm must be greater than 0.2645 , a numerical computation. In that case, the major axis of the forearm must be less than 0.2355 , another computation. In order that the distance the forearm must span be less than 0.2355 , the point $d$ must be further than $\frac{1}{5}$ from the $y$-axis. If $d$ is further than $\frac{1}{5}$ from the $y$-axis, then the point of contact between $\Gamma$ and the "bottom" of the inverted cover is on the upper arm and is above the major axis of the upper arm. With this point of contact, rotating $\Gamma$ clockwise about $m$ allows $m$ to move up, not down, until $\alpha$ and $\gamma$ both touch, contradicting MPS.

Case 2B. Finally, consider the case where, raising the worm into the inverted cover $C^{* *}, \alpha$ touches first. If, before we raised it, $\alpha$ as well as $\gamma$ escaped, then the argument in Case 2A goes through essentially unchanged, except that we rotate counterclockwise instead of clockwise. If, on the other hand, $\alpha$ was covered by the original cover in the original MPS position, then the point on $\alpha$ that touches must be further from the $y$-axis than $d$, because the distance between the top of $C^{*}$ and the "bottom" of $C^{* *}$ decreases as the distance from the $y$-axis increases. Also, the point on $\alpha$ that touches must lie to the left of the $y$-axis, since $e$ lies to the left of $c$. Then a similar argument to the one in Case $2 \mathrm{~A}$ shows that by rotating the worm counterclockwise, keeping $\alpha$ just touching the "bottom" of $C^{* *}$, we can bring $\gamma$ into contact with the "bottom" of $C^{* *}$ while keeping $m$ above the origin, contradicting MPS.

Therefore, in every case the worm that satisfies MPS is covered. This completes the proof.

\section{Closing Remarks}

In adding the "bubbles" (small portions of the reflection of the top boundary of $C$ in the $x$-axis) to the bottom boundary $\beta$ of region $C$ to obtain $C^{*}$, we only added a small amount of area, approximately 0.0007765 . However, it facilitated the proof. We strongly believe 
the original constructed cover $C(t=0.1527985)$ with area 0.2596605 is a cover. In our construction of $C^{*}$ we used non-convexity to reduce the area of the enclosed region, not to facilitate the proof. The overall left-right design on $C^{*}$ was used to facilitate the proof.

If the region $C(t)$ were constructed to maintain convexity by using a straight boundary from $(0, b)$ to $\left(\frac{1}{2}, 0\right)$ rather than a parabolic boundary, the minimum value of $t$ (to keep the worm from escaping via the top-bottom order while the center $m$ remains at or below the origin) occurs at $t=0.13505$ with $b=-0.36495$. In this case the proof goes through as above (no bubbles required). Moreover, the area of the resulting convex cover is 0.2738086 which is also less than the current "record" of 0.2752373 [10]. Finally, the region $C^{*}$ can be used to reduce the volume of existing covers in higher dimensions for multidimensional worms [4].

\section{Acknowledgment}

We are grateful to the referees, whose comments and suggestions improved the presentation and readability of this paper.

\section{References}

1. A. S. Besicovitch, On arcs that cannot be covered by an open equilateral triangle of side 1, Math. Gaz. 49 (1965), 286-288; MR 32 \#6320.

2. S. Finch, Website http://pauillac.inria.fr/algo/bsolve/constant/worm/worm.html, MathSoft Engineering and Education, Inc.

3. J. Gerriets and G. Poole, Convex regions which cover arcs of constant length, Amer. Math. Monthly 81 (1974), 36-41; MR 48 \#12310.

4. J. Håstad, S. Linusson, and J. Wästlund, A smaller sleeping bag for a baby snake, Discrete Comput. Geom. 26 (2001), 173-181; MR 1832735 (electronic).

5. M. Laidacker and G. Poole, On the existence of minimal covers for families of closed bounded convex sets, Unpublished, 1986.

6. J. M. Marstrand, Packing smooth curves in $R^{q}$, Mathematica 26 (1979), 1-12; MR 81d:52009.

7. L. Moser, Poorly formulated unsolved problems of combinatorial geometry, Mimeographed list (1966), possibly reported at the Combinatorial Geometry Conference at East Lansing, March 1966.

8. W. Moser, G. Blind, V. Klee, C. Rousseau, J. Goodman, B. Monson, J. Wetzel, L. M. Kelly, G. Purdy, and J. Wilker, Fifth edition, Problems in Discrete Geometry. McGill University, Montreal, 1980; MR $84 \mathrm{c}: 51003$.

9. W. Moser and J. Pach, Recent Developments in Combinatorial Geometry: New Trends in Discrete and Computational Geometry. Algorithms Combin., 10, Springer-Verlag, Berlin, 1993; MR 94f:52032.

10. R. Norwood, G. Poole, and M. Laidacker, The worm problem of Leo Moser, Discrete Comput. Geom. 7 (1992), 153-162; MR 92j:52014.

11. G. Poole and J. Gerriets, Minimum covers for arcs of constant length, Bull. Amer. Math. Soc. 79 (1973), 462-463; MR 47 \#4150.

12. H. Seifert and W. Threlfall, A Textbook of Topology, Academic Press, New York, 1980.

13. J. Schaer, The broadest curve of length 1, Research paper 52, University of Calgary, 1968.

14. J. E. Wetzel, On Moser's problem of accommodating closed curves in triangles, Elem. Math. 27 (1972), 35-36; MR 45 \#4282.

15. J. E. Wetzel, Sectorial covers for curves of constant length, Canad. Math. Bull. 16 (1973), 367-375; MR $50 \# 14451$.

Received January 22, 2002, and in revised form September 11, 2002. Online publication January 30, 2003. 